\title{
The importance of being expert: Top-down attentional control in visual search with photographs
}

\author{
Orit Hershler ANd Shaul Hochstein \\ Hebrew University, Jerusalem, Israel
}

\begin{abstract}
Two observers looking at the same picture may not see the same thing. To avoid sensory overload, visual information is actively selected for further processing by bottom-up processes, originating within the visual image, and top-down processes, reflecting the motivation and past experiences of the observer. The latter processes could grant categories of significance to the observer a permanent attentional advantage. Nevertheless, evidence for a generalized top-down advantage for specific categories has been limited. In this study, bird and car experts searched for face, car, or bird photographs in a heterogeneous display of photographs of real objects. Bottom-up influences were ruled out by presenting both groups of experts with identical displays. Faces and targets of expertise had a clear advantage over novice targets, indicating a permanent top-down preference for favored categories. A novel type of analysis of reaction times over the visual field suggests that the advantage for expert objects is achieved by broader detection windows, allowing observers to scan greater parts of the visual field for the presence of favored targets during each fixation.
\end{abstract}

Visual scenes contain an enormous amount of information. Not all of the information present in a visual scene can be processed simultaneously. Rather, the visual system selectively chooses which parts or characteristics of the scene are the most important or the most relevant for the task at hand at any point in time, and these are processed first. An accumulating body of evidence indicates that two different processes influence the deployment of visual attention within a visual scene: a bottom-up mechanism using cues present in the visual scene itself, and a top-down mechanism driven by factors depending on the observer (Egeth \& Yantis, 1997; Itti \& Koch, 2000). It should be noted that factors considered to originate in the visual scene are not necessarily processed at the lower levels of the visual hierarchy. Rather, it has been suggested, on theoretical and empirical grounds, that the first explicit effect of external, bottom-up cues may be at higher and more conceptual levels of the visual hierarchy, before further, slower, more detail-dependent processing occurs at lower visual levels (Ahissar \& Hochstein, 1997, 2004; Hochstein \& Ahissar, 2002).

Bottom-up control of visual attention is usually understood in terms of salience, the relative difference between a region in the visual scene and its surroundings in terms of low-level factors such as color, contrast, texture, and orientation. Generally, salience models assume the existence of a salience map that encodes the salience of each part of a visual scene (Koch \& Ullman, 1985). This master map controls visual attention through a process of neural competition, deploying attention to the most salient location at any point in space.

In contrast, by definition, top-down control of visual attention depends on interactions among the observer, the scene, and the task. For this reason, top-down attentional control is more variable than low-level control. Despite these difficulties, several models for the implementation of top-down attentional control have been suggested, frequently incorporated in salience-type models. For example, Cave and Wolfe (1990), in the guided search model, suggested that in visual search, the top-down component for the effective salience of each location within a particular feature map is the degree of similarity of the feature at that location and the target value for that feature dimension. Top-down attentional control may also influence competition among early visual filters (Lee, Itti, Koch, \& Braun, 1999) or interact with salience by shunting inhibition-of-return mechanisms (Itti \& Koch, 2000, 2001). In general, both bottom-up and top-down influences on attentional control can be tested through the visual search paradigm. Wolfe, Butcher, Lee, and Hyle (2003) investigated the effects of top-down and bottom-up influences on singleton search, finding that uncertainty about target identity, a form of top-down information, slows down search. Bottom-up guidance still supported efficient search under these conditions.

Top-down attentional control indicates that certain parts of the visual field are more interesting than other parts, complementing bottom-up cues. We suggest that as part of

O.Hershler, oritaron@gmail.com 
this process, objects that belong to categories that are important or special to the observer may be given additional salience through top-down control. One example of such a top-down influence is stimulus familiarity, which has been studied extensively in visual search. Research has shown that, surprisingly, search is most efficient for unfamiliar targets on familiar distractors (Wang, Cavanagh, \& Green, 1994), whereas efficient search for familiar targets occurs on familiar distractors only (Malinowski \& Hübner, 2001). A study with naturalistic objects also showed that visual experience facilitates processing of both target and distractor items during search (Mruczek $\&$ Sheinberg, 2005). However, search speed for familiar, trained items did not generalize to other, unfamiliar targets of the same category.

Studies on the familiarity of targets and distractors have traditionally focused on homogeneous displays and relatively simple search items - for example, mirror-image $N \mathrm{~s}$ and regular $N \mathrm{~s}$ for speakers of German and Slavic (only the latter are familiar with the mirror image; Malinowski \& Hübner, 2001). In these studies, stimulus familiarity is confined to repetitions of a single exemplar. We asked whether the familiarity effect would extend to entire categories, encompassing many different exemplars, and tested the hypothesis that top-down salience may be extended to entire categories by looking at the performance of experts on a visual search task.

We asked car experts and bird experts to detect cars and birds among heterogeneous distractors. Both groups of experts viewed exactly the same stimuli, so that any bottomup processing effects would be identical and the top-down influence of expertise could be assessed in isolation.

Studies on visual expertise indicate that experts process objects of their expert category in a more holistic fashion (Gauthier \& Tarr, 2002) and parse these objects differently than do novices (reviewed in Palmeri, Wong, $\&$ Gauthier, 2004) and that the entry point of expert object recognition shifts from the basic to the subordinate level (Tanaka \& Taylor, 1991; see Rosch, Mervis, Gray, Johnson, \& Boyes-Braem, 1976). The latter finding has come to be recognized as one of the hallmarks of expertise (Palmeri et al., 2004). The effect of expertise on visual category search, which does not require subordinate classification, has not yet been examined, and the present study is unique in this respect.

The human face is an example of a potentially important category that could receive top-down salience. Indeed, Hershler and Hochstein (2005) reported rapid popout in visual search for natural face photographs among heterogeneous distractor photographs, with a near parallel search reaction time (RT) slope over increasing set sizes (Treisman \& Gelade, 1980). This rapid search with nearzero slope was not observed for other categories, such as cars, flowers, animals, or animal faces. Due to the great variation of distractors and face targets used in the study, the face search appeared not to rely on a single basic lowlevel feature. Rather, an effective search mechanism would have to rely on the higher level categorical concept of face versus nonface. This mechanism may be implemented through a special face feature salience map, based on the unification of a number of low- and high-level features characteristic of faces (Hershler \& Hochstein, 2005).

Faces are distinguished from other object categories by top-down influences such as social significance or expertise. Indeed, face-specific effects such as face inversion (Farah, Tanaka, \& Drain, 1995) or prosopagnosia (Whiteley \& Warrington, 1977) were found with other homogeneous categories of expertise (Levine \& Calvanio, 1989). Effects that appear to be face specific should, therefore, be compared with nonface stimuli that match the constraints found for faces (Gauthier \& Tarr, 1997). A second goal of the present study was to compare search for faces with search for objects of expertise, to ascertain what role human expertise for faces might play in the face search advantage. If objects of expertise have no search advantage, it could be claimed that face search relies on low-level factors that are present only for faces (as was suggested by VanRullen, 2006). On the other hand, if there is a general search advantage for objects of expertise, depending on each person's particular expertise, we would have to conclude that the expert search advantage cannot rely only on bottom-up stimulus properties, and most parsimoniously, the same would apply to faces.

\section{METHOD}

\section{Subjects}

Eleven subjects participated in the experiment. Of these, 6 were bird experts and 5 were car experts. Each subject participated in all three target conditions of the experiment: search for faces, search for birds, and search for cars. Bird experts belonged to a bird-watching center and ranged from 17 to 34 years of age; car experts either worked with cars or were highly interested in car models and ranged from 18 to 39 years of age. All the subjects had normal or correctedto-normal vision. We tested their ability to identify 50 different birds or cars; bird experts averaged 37 correct answers, and car experts averaged 34 correct answers on these difficult recall tests. By comparison, 4 novices in both fields averaged 5 correct answers on the bird recall test and 4 correct answers on the car recall test.

\section{Stimuli}

The stimuli were presented on the screen of an IBM T43 laptop computer set at $60-\mathrm{Hz}$ refresh and a resolution of $1,024 \times 768$ pixels; the subjects sat at a viewing distance of approximately $40 \mathrm{~cm}$. The display consisted of arrays of $3 * 3,4 * 4,5 * 5$, or $6 * 6$ photographs, as shown in Figure 1, created by an in-house software package from color photographs of 84 birds, 84 faces, 70 cars, and 261 color photographs of other objects as distractors. All the displays were centered on the midpoint of the screen, the location of the fixation cross. Each element of the array subtended $4^{\circ}$ (width) $\times$ $3.8^{\circ}$ (height) of visual angle, with an additional average distance between elements of $1.25^{\circ}$ (width) and $0.25^{\circ}$ (height). Random jitter was introduced to disturb alignment of the elements; maximum jitter was $0.2^{\circ}$ (vertical) and $0.5^{\circ}$ (horizontal). For each target condition, half of the stimuli contained the pre-designated target (face, bird, or car), and half did not contain the target. In all the target-present stimuli, only one target category was present. Targets were pseudorandomly placed at each location in the search array.

\section{Experimental Procedure}

The experiment was divided into three blocks with a single target type in each block - face, car, or bird. Each block contained 280 trials, presented semirandomly so that all set sizes, target positions, and target presence/absence conditions were equally divided in each block. The subjects were given the option of taking small 5-min 


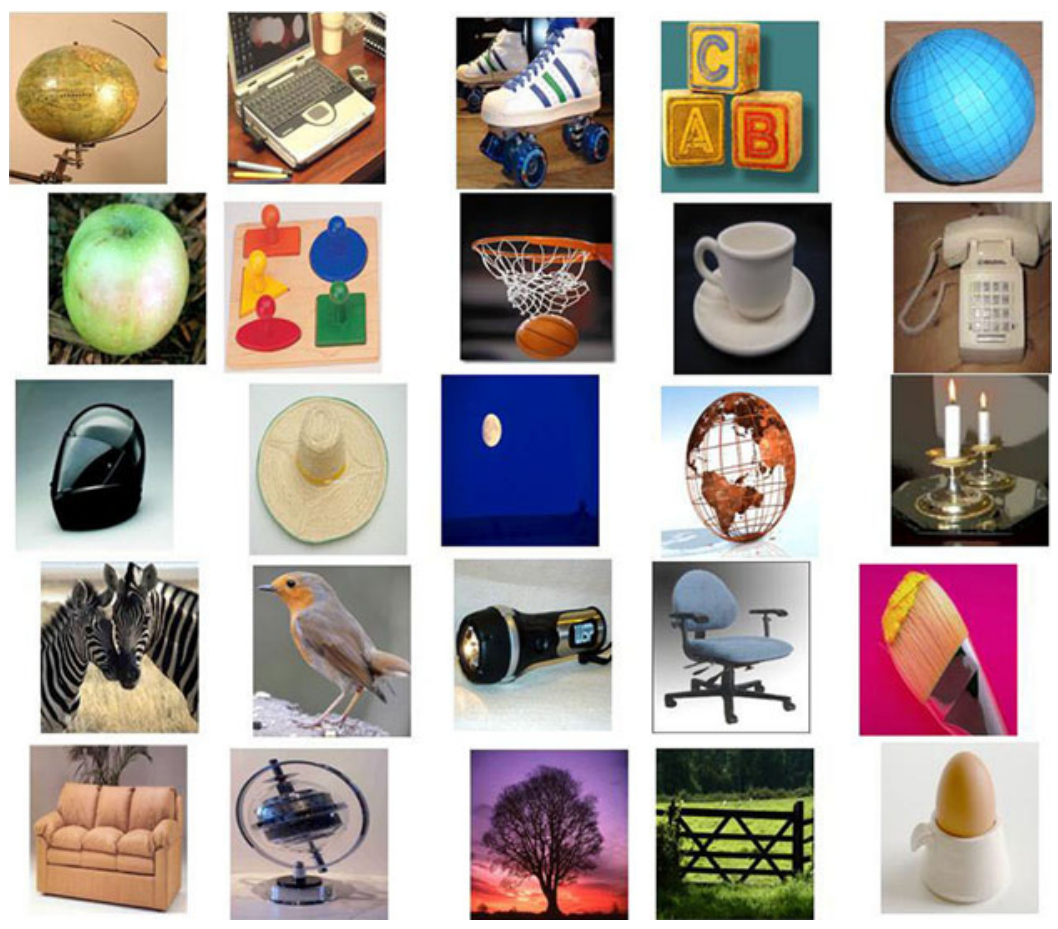

Figure 1. Example stimulus. The stimuli contained 9, 16, 25 (shown), or 36 elements. The targets were bird (shown), car, or face photographs.

breaks between blocks. In each block, the subject was given both printed and oral instructions about which target to look for. The order of the blocks was counterbalanced across subjects and type of expertise, so that both car and bird experts performed the experiment in every possible order.

The subjects were instructed to view the stimuli and report as accurately and as quickly as possible whether they contained the prespecified target category. Between trials, a fixation cross appeared in the center of the screen. The search stimulus remained on the screen until the subject made his or her response by means of the mouse (right mouse click for target present, left mouse click for target absent), and RT was measured (there was no need to place the cursor on the target). A computer-generated tone provided negative feedback after errors, to enable the subjects to monitor their accuracy levels. All the subjects gave their informed consent to participate in the experiment and were paid for participation.

\section{RESULTS}

\section{Search for Expert and Novice Target Categories}

The first goal of this experiment was to compare search times for both car and bird experts when searching for car or bird targets. The average RT and accuracy for the two groups of experts on the search for cars and birds when the targets were present is shown in Figure 2, top. RT slopes for both present and absent trials are shown in Figure 2, bottom. Figure 3 shows individual data for all the subjects for target-present and target-absent trials.

We analyzed target-present and target-absent trials separately. To ensure that there was no large difference in the search abilities of the two groups of subjects or the a priori salience of the two sets of images, we performed a repeated measures ANOVA on RT and accuracy for target- present trials. The ANOVA revealed no significant main effect of target type on accuracy and no main effects of type of expertise on RT or accuracy. Target type did have a significant influence on search speed, indicating that cars were slightly easier to find than birds; averaging over the two groups of experts, RTs were $1,185 \mathrm{msec}$ for cars and $1,374 \mathrm{msec}$ for birds $[F(1,9)=8.80, p<.05]$. Accuracy when the data for the two groups were combined was $90 \%$ for cars and $87 \%$ for birds $[F(1,9)=5.86, p=$ .39]. Similarly, there was no difference between the two groups of experts when their data for both target types were combined: RT was 1,340 msec for car experts and $1,229 \mathrm{msec}$ for bird experts $[F(1,9)=0.92, p=.36]$, and accuracy was $88 \%$ for car experts and $90 \%$ for bird experts $[F(1,9)=0.42, p=.53]$. This means that the two expert groups were matched in terms of their overall search abilities and that cars were only slightly more detectable than birds.

On the other hand, the interaction between type of expertise and target category for target-present trials was highly significant, for both RT and accuracy, as displayed in Figure 2, top [RT, $F(1,9)=25.62, p<.01$; accuracy, $F(1,9)=16.29, p<.01]$. This suggested that expertise and target category might have effects when each group was analyzed separately. Paired $t$ tests on each group of experts separately indicated that the car experts were faster $[t(5)=$ $-4.24, p<.01]$ and more accurate $[t(5)=4.26, p<.01]$ when looking for cars than when looking for birds. The bird experts were also significantly faster $[t(5)=2.23, p<$ $.05]$ but not significantly more accurate $[t(5)=-1.22$, $p=.14]$ when looking for birds than when looking for 


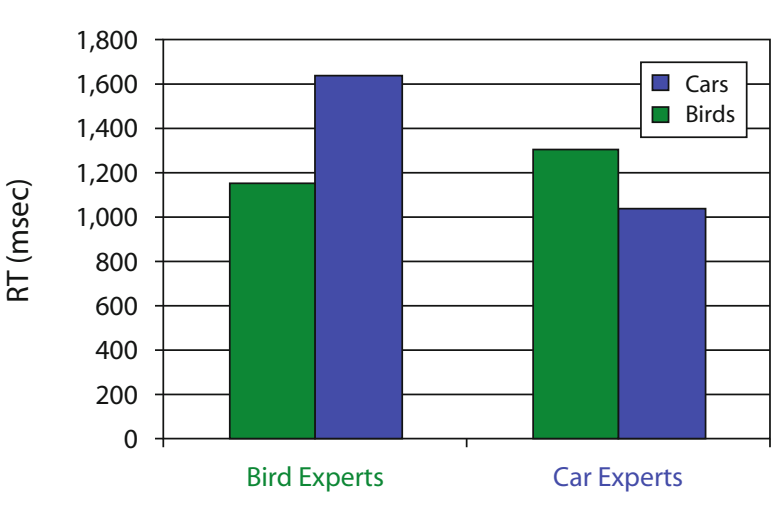

Car Experts

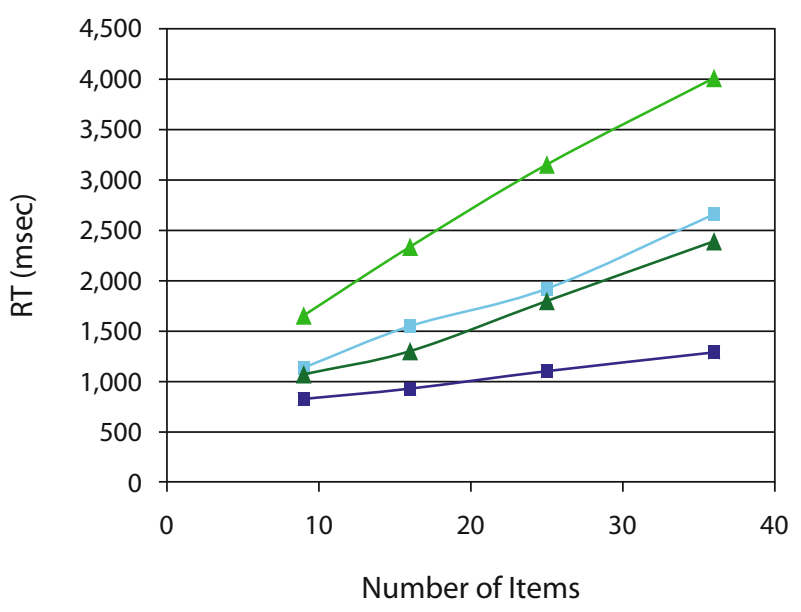

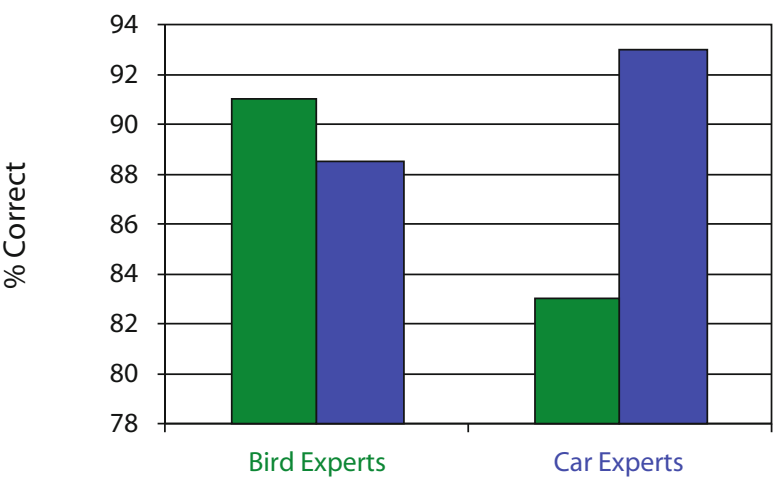

Bird Experts

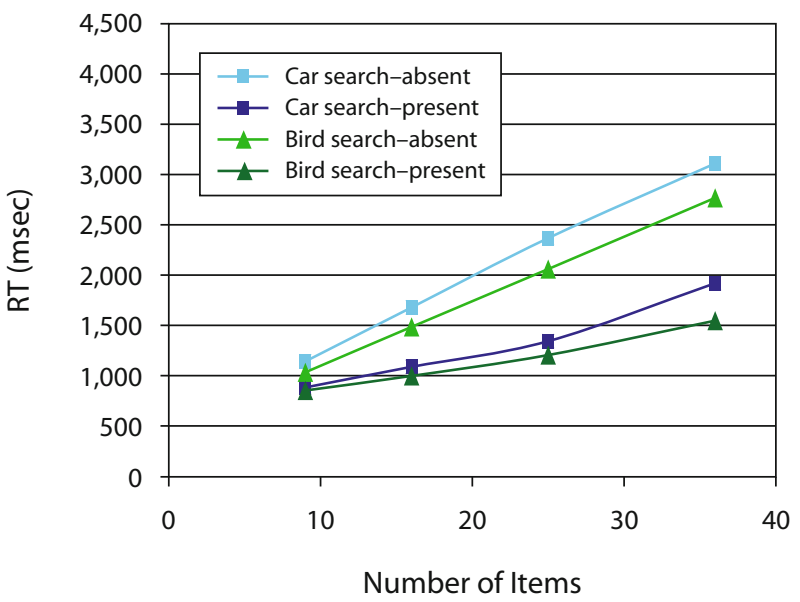

Figure 2. Top: Average reaction times (RTs; left) and percentages of correct responses (right) as a function of expertise type and target category, target-present trials. Bottom: RTs as a function of set size for car experts (left) and bird experts (right).

cars. For all target types, the experts were significantly faster (but not significantly different in accuracy) than their nonexpert peers, as follows: Paired $t$ tests on target categories revealed that the car experts were faster $[t(9)=$ $7.16, p<.05]$ but not significantly more accurate $[t(9)=$ $2.49, p=.14]$ than the bird experts when looking for cars, whereas the bird experts were faster $[t(9)=8.58, p<.05]$ but not significantly more accurate $[t(9)=4.38, p=.07]$ than the car experts when looking for birds.

RT slopes for the category of expertise for target-present trials were markedly lower than those for the novice category (Figure 3, top right), although it should be noted that slopes did not approximate parallel search slopes. Slopes were lower when the car experts were finding cars than when they were finding birds $[t(4)=2.13, p<.05]$, whereas slopes were lower when the bird experts were finding birds than when they were finding cars $[t(5)=$ 2.01, $p<.01]$. In addition, the experts had lower search slopes for their expert category than did the novices; there was a significant effect of expertise on search slope for cars $[t(9)=1.83, p<.01]$, as well as a less significant effect on search slopes for birds $[t(9)=2.13, p<.05$, $t$ test assuming inequality of variances], as revealed by
Levene's test for the variances of the bird search RT slopes $[F(1,9)=16.80, p<.01]$.

Overall, these results indicate that perceptual expertise influenced speed, accuracy, and search slopes for visual search: The experts were faster and more accurate at detecting their expert category. In addition, when given the same displays, the car experts detected cars more quickly and with lower search slopes than did the bird experts, whereas the bird experts detected birds more quickly and with lower slopes than did the car experts.

We performed a separate analysis on the data from target-absent trials (Figure 3, bottom). Results indicated that the car experts detected the absence of birds significantly more slowly [mean RT for cars $=1,818 \mathrm{msec}$, mean RT for birds $=2,790 \mathrm{msec} ; t(4)=2.13, p<.01]$ and with larger search slopes [slope for cars $=57 \mathrm{msec} /$ item, slope for birds $=87 \mathrm{msec} /$ item, $t(4)=2.13, p<$ $.05]$ than they detected the absence of cars, but these effects were not observed for the bird experts. The bird experts were faster than the car experts in detecting the absence of birds [mean RT for bird experts $=1,838 \mathrm{msec}$, mean RT for car experts $=2,790 \mathrm{msec} ; t(9)=1.83, p<$ $.05]$, but the car experts were not significantly faster than 
RT
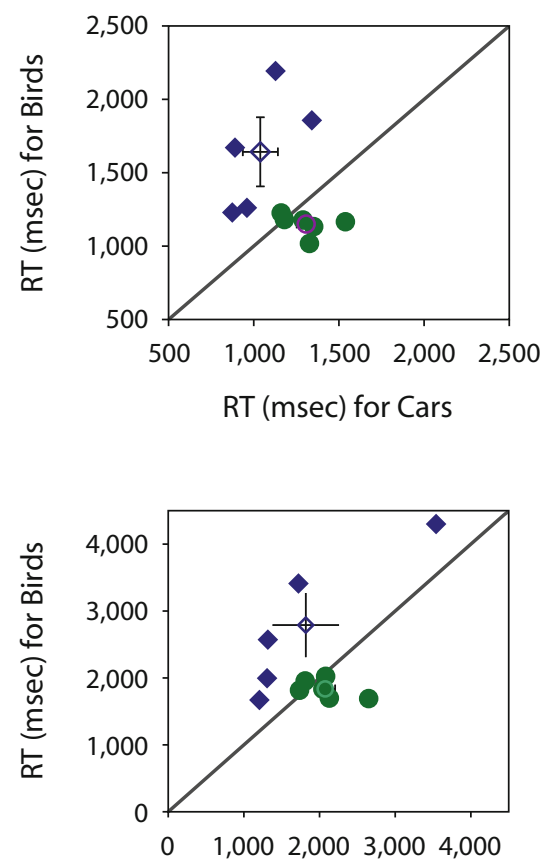

RT (msec) for Cars

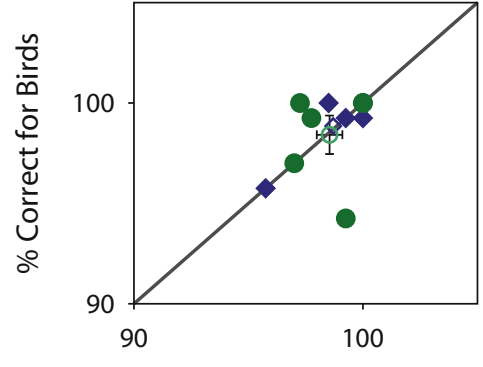

$\%$ Correct for Cars
Search Slopes
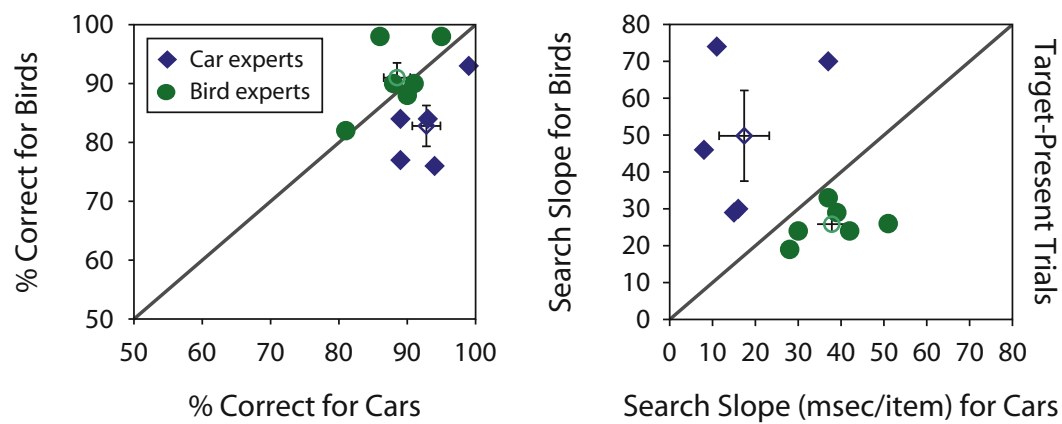

Search Slope (msec/item) for Cars

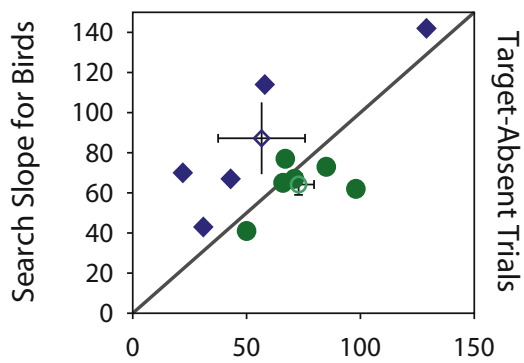

Search Slope (msec/item) for Cars

Figure 3. Top: Individual subject data for target-present trials, showing average reaction times (RTs; left), accuracy (center), and search slopes (right) in search for birds (ordinate) and cars (abscissa). Bottom: Individual data for target-absent trials. Bird experts (filled circles) were faster and more accurate and had lower search slopes when searching for a bird than when searching for a car; car experts (diamonds) had the opposite trends. Means and standard errors are indicated by open circles and error bars.

the bird experts in detecting the absence of cars. These results probably reflect the fact that the cars were slightly more detectable than the birds (see above), enabling all the subjects, regardless of expertise, to more rapidly mark the absence of cars than the absence of birds. There were no other significant differences in RT slopes or accuracies for the target-absent data across the two groups of experts. Thus, fewer significant effects were found in the targetabsent data than in the target-present data.

\section{Comparison With Search for Faces}

The second goal of this experiment was a comparison of search performance for objects of expertise with that for faces. A statistical analysis was performed with both target types collapsed into an expert or nonexpert category for all subjects. This revealed a significant main effect of target type (expert category, nonexpert category, and faces) on RT $[F(2,20)=35.32, p<.001]$ and accuracy $[F(2,20)=19.68, p<.001]$. Further analysis showed all pairwise comparisons to be significant, with search for faces being found to be both faster and more accurate than that for the expert category $(p<.05)$, and search for the expert category being found to be faster and more accurate than that for the nonexpert category $(p<.05)$. Search slope analysis followed the same pattern, with a significant main effect for target type $[F(2,20)=27.37$, $p<.001]$, and significance for all pairwise comparisons (face vs. expert, $p<.01$; expert vs. novice, $p<.01$ ]. In summary, these results show that search performance data for faces, expert targets, and control targets lie along a continuum for each of the measures of speed, accuracy, and search slope (Figure 4).

\section{Target Salience Over the Visual Field}

To assess how the search advantage for certain target categories is achieved, we analyzed target salience over the visual field. Search times for target-present trials were analyzed as a function of target position for the three target categories, with bird and car targets collapsed into expert and novice categories. Results (for set size 36) are shown in Figure 5, top. Intervening values were created by MATLAB bilinear interpolation. The bottom graph in Figure 5 is a cumulative histogram of these data (full circles), showing the number of positions (out of a possible total of 36) at which targets were detected as a function of RT. Note that the curve for experts - and even more so, that for facesrises faster and has a higher maximal level. Performance for smaller set sizes followed the same pattern.

The data of the three curves in Figure 5 (bottom) can be approximated by the exponential approach function:

$$
N=N_{\max }-N_{\max } * e^{-k\left(t-t_{0}\right)},
$$

where $N$ is the number of detected target positions, $t$ is time in milliseconds, $N_{\max }$ is the maximum number of items accurately detected, $k$ is a slope coefficient, and $t_{0}$ is a constant related to the minimum time needed to detect an item at fixation. Fitted curves are shown as lines in Figure 5 (bottom). Table 1 presents the fitting parameters 

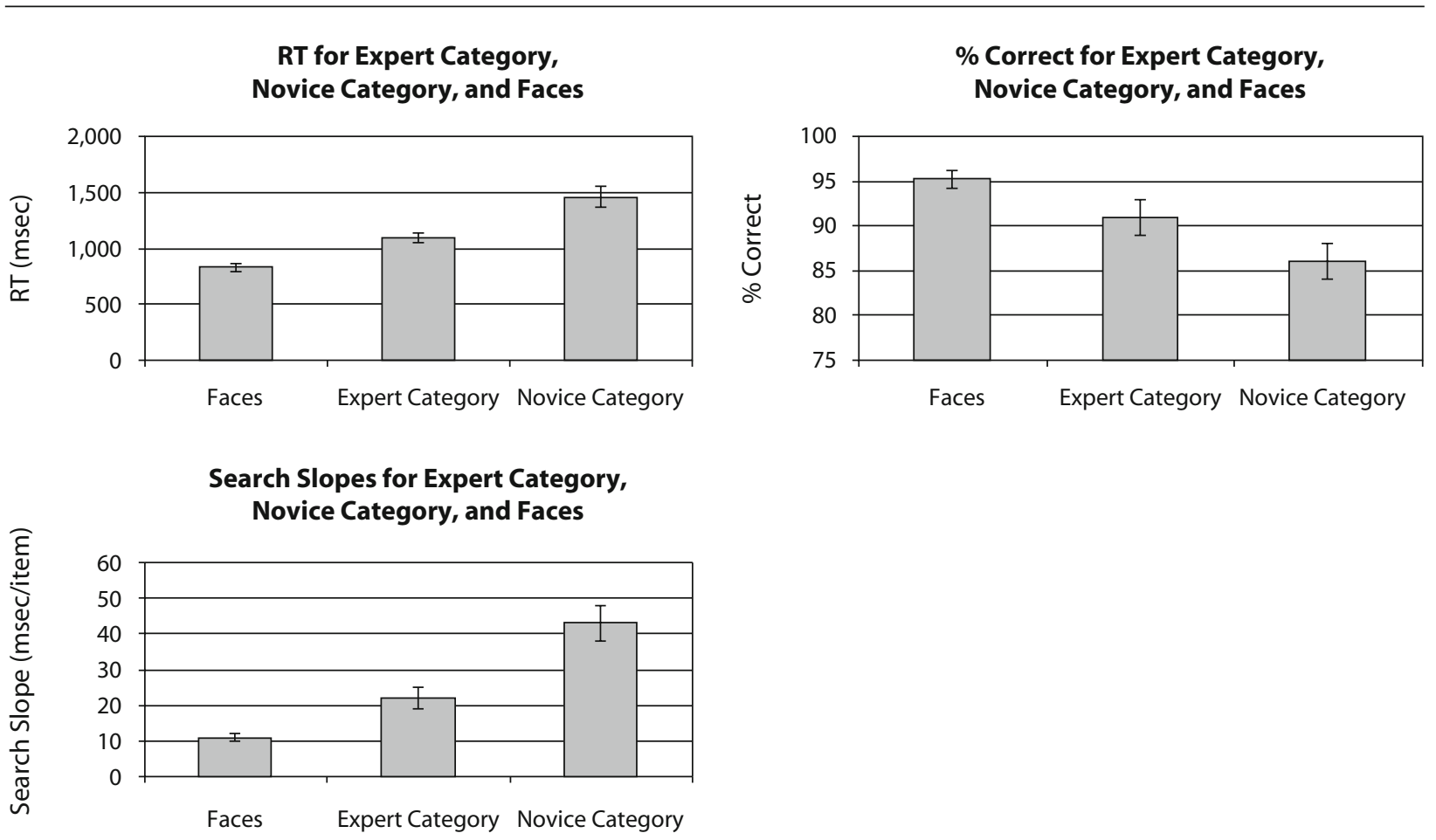

Figure 4. Reaction times (RTs), percentages of correct responses, and search slopes during search for an exemplar of the expert category (birds or cars), novice category (cars or birds), and faces. Note that data for the category of expertise are in between those for faces and those for the category without expertise.

for search for faces, expert targets, and control targets. To calculate the slope for each curve, we calculated the derivative of the exponential approach function:

$$
d N / d t=\left(N_{\max } * k\right) * e^{-k\left(t-t_{0}\right)} .
$$

The initial and maximum slope of each curve, $N_{\max } * k$, is obtained by substituting $t_{0}$ for $t$ in the derivative. Calculated maximum slopes are shown in Table 1.

Figure 5 indicates that, on average, targets close to the fixation point were detected more quickly, with average detection times progressively slowing toward the periphery, replicating previous findings (Carrasco, McLean, Katz, \& Frieder, 1998). In addition, the number of elements detected at shorter RTs were highest for faces, smaller for objects of expertise, and smallest for control objects. To quantify this difference in the effective size of the detection window, we calculated a variant of the fullwidth-at-half-height (FWHH) measure. First, we created cumulative histograms for each subject and each target category (similar to the average histogram in Figure 5, bottom). We then defined the longest RT for each subject as the time when he or she reached the maximal level of detected items for that category (i.e., if a subject detected only 32 of the novice targets, the time at which the 32nd target was detected was the longest RT). The FWHH is the number of targets that were detected with RTs shorter than or equal to half the above-defined longest RT for that target category. This yielded an average of 30 items $( \pm 0.8)$ for faces, 26 items $( \pm 1.3)$ for the expert targets, and 20 items $( \pm 1.5)$ for the novice category. A repeated measures
ANOVA on this variant of the FWHH showed that this difference was significant $[F(2,30)=19.00, p<.001]$, with pairwise comparisons significant as well (faces vs. expert, $p<.01$; expert vs. novice, $p<.01$ ). This result suggests that the window of detection grew wider for the experts when they were searching their favored categories, consistent with previous findings on greater expert visual spans (Reingold, Charness, Pomplun, \& Stampe, 2001). The data are consistent with a search mechanism that detects the presence or absence of target items in groups of elements, rather than through single fixations. The advantage of this search window is highest for faces, intermediate for expert targets, and smallest for control targets. As search progresses, the detection window becomes less effective, probably due to an increasing amount of overlap of the search window with positions that were already scanned.

\section{DISCUSSION}

In this study, two groups of experts viewed exactly the same stimuli but reacted differently according to their prior experience and personal expertise. Targets from each category were detected considerably more efficiently by experts for that category. The more efficient visual search by experts can be accounted for only by top-down influences, such as familiarity with the search targets. In counterintuitive fashion, visual search studies with homogeneous targets and distractors have shown that search appears to be most efficient when distractors are familiar and the target novel (e.g., Rauschenberger \& Chu, 2006; 


\section{Detected Target Positions as a Function of Time}

Face Search

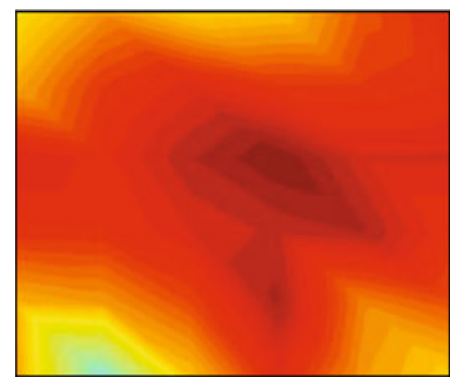

Expert Search

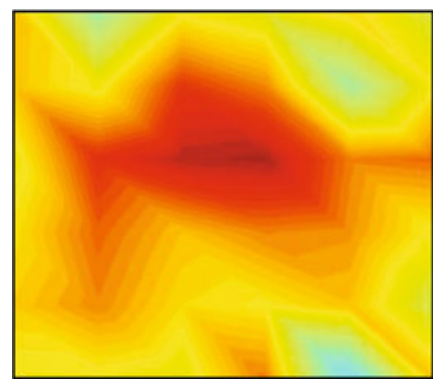

Novice Search

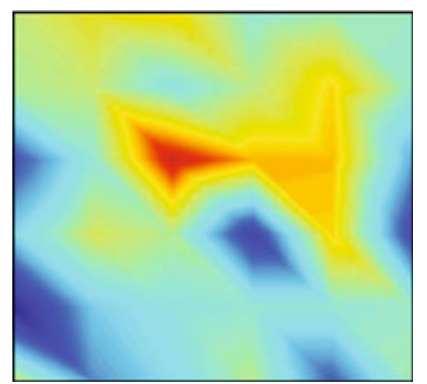

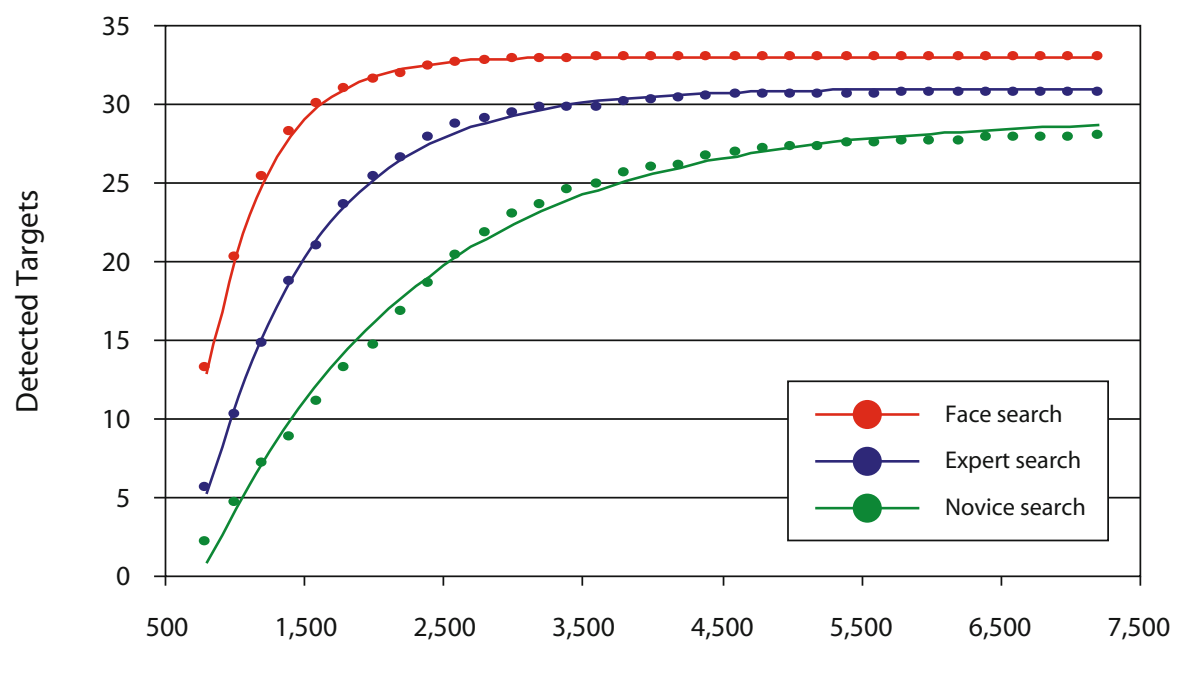

RT

Figure 5. Top: Average search times (in milliseconds) for target-present trials as a function of target position for each of the three target categories, for set size 36; that is, target could appear at any one of 36 positions. The top left of the graphs corresponds to the top left position in the search array. Interleaved values determined by MATLAB bilinear interpolation. Bottom: Cumulative histogram of average number of detected target positions as a function of reaction time. Curves are best-fit exponentials to averaged data points.

Shen \& Reingold, 2001; Wang et al., 1994). On the other hand, in line with our results, Mruczek and Sheinberg (2005) studied familiarity with heterogeneous displays of real objects and found that under certain conditions, familiar targets become more salient than novel targets. This salience failed to generalize to previously unseen exemplars of the same category, since the subjects were not category experts. The present study expanded on these results by testing the salience of entire expert categories, even for previously unseen specific exemplars.

The second goal of the study was a comparison of face search with search for expert objects. Search efficiency was highest for faces, intermediate for expert objects, and lowest for regular objects. Importantly, the advantages for cars and birds were found only for people who were experts in the relevant category and not for those who were expert in the other category. The fact that different types of experts searched the same displays controlled for any potential confound that might arise from birds or cars being more or less salient due solely to their bottom-up stimulus properties. Rather, it must have been a top-down learned effect that allowed exemplars of specific categories to be found more quickly by some people than by others. Does this conclusion that search is speeded by top-down expertise generalize to faces? Since faces are considered to be a category of expertise for normal human subjects, the top-down holistic factor of expertise is likely to play a role in the rapid visual search for faces as well. The pres-

Table 1

Calculated Slope Coefficients

\begin{tabular}{lccc}
\hline & $\begin{array}{c}\text { Face Target } \\
\text { Search }\end{array}$ & $\begin{array}{c}\text { Expert Target } \\
\text { Search }\end{array}$ & $\begin{array}{c}\text { Control Target } \\
\text { Search }\end{array}$ \\
\hline$N_{\max }($ items $)$ & 33.02 & 30.98 & 29.11 \\
$k\left(\mathrm{msec}^{-1}\right)$ & 0.0023 & 0.0013 & 0.0007 \\
$t_{0}(\mathrm{msec})$ & 585.1 & 652.0 & 757.0 \\
$N_{\max } * k($ items $/ \mathrm{msec})$ & 0.08 & 0.05 & 0.02 \\
$R^{2}(\%)$ & 99.89 & 99.62 & 99.24 \\
\hline
\end{tabular}


ent results make it unlikely that the face search advantage is caused by a single low-level factor alone (VanRullen, 2006). Rather, bottom-up attributes of faces interact with top-down factors, such as expertise, to cause the face search advantage (Hershler \& Hochstein, 2005, 2006). The locus of such an interaction could be a high-level, holistic, view-invariant expert template that receives information about many different, lower level attributes and combines these into a single percept. Interestingly, Tong and Nakayama (1999) reported faster search for one's own face than for a stranger's face, which persisted even after hundreds of presentations of the unfamiliar face. They concluded, among other things, that the own-face advantage requires extensive experience to develop and is likely to include some sort of abstract, view-invariant information. The early appearance of face expertise and a preference for observing faces would indicate some inherentgenetically transferred-expertise.

What is it that experts can do but novices cannot? One possibility is that expert subjects have more effective "guiding" templates for their preferred category than for their nonpreferred category, enabling experts to separate images into potential targets and nontargets with spread attention, effectively decreasing set size. These holistic templates could support two different processes: a process by which doubtful images are discarded as potential targets, and another by which promising images are entered into the potential target set. This could also explain the difference between the target-present and target-absent data: For the target-present case, both processes are effective, whereas in target-absent trials, only the discarding process is useful. The use of two effective expert mechanisms in target-present trials versus only one for target-absent trials could explain the smaller differences between experts and novices in target-absent trials. It should be noted that experts are also likely to have greater motivation to find expert targets, which could influence search speed. Further research is needed to elucidate this issue.

These results are especially interesting since expertise has traditionally been seen as involving superior subordinate categorization (reviewed in Palmeri et al., 2004; but see Johnson \& Mervis, 1997), along with more efficient parsing (Goldstone, 2003) and holistic processing (Gauthier, Curran, Curby, \& Collins, 2003). The present results add another ability to the growing list: Experts are able to find objects of their expertise more quickly than can novices. This hints at experts' having both distinguishing and detection skills and, perhaps, that these skills go together. The "bird template" in bird experts might be more developed, so that it enhances the ability both to distinguish among birds and to find them. Subordinate categorization involves the ability to distinguish between different exemplars from the expert category, whereas object search among distractors seems to rely on a different ability. A superior basic-level category representation for experts could explain the search advantage, but it is not clear how this relates to the accepted superior-subordinate-level category representation for experts. For example, a "bird search template" would have to be effective over large areas of the visual field, whereas a "bird distinguishing template" would presumably be effective mostly at the fovea (for the simple reason that some distinctions between bird species are relatively minute).

How is the search advantage for faces and objects of expertise achieved? Two different mechanisms are likely to be responsible. First, our data indicate a spatial advantage for the favored category, enabling subjects to fixate on one point in space and detect the presence of the favored category in the surrounding group. This may be achieved by a peripheral detection mechanism or the ability to check for target presence in a group of distractors simultaneously. Second, the shorter search times for objects of expertise at the center of the search display indicate that these objects are detected faster at fixation as well, enabling a greater number of, and more rapid, fixations. Favored targets might also be detected during saccadic eye movements, by selective sparing of saccadic suppression (Burr, Morrone, \& Ross, 1994), although the speed of saccades and the nature of the sparing mechanism make this option unlikely. Search for faces and for targets of the category of subject expertise was more efficient in two ways: Detection time was shorter for favored targets presented at fixation, and this ability for faster detection was present for a wider visual field, as quantified by the number of items detected at half the longest RT for that category. For faces especially, the coverage of the visual field is quite rapid; face presence is detected in $70 \%$ of the field $400 \mathrm{msec}$ after it is detected at fixation, and $94 \%$ of the visual field is covered after $600 \mathrm{msec}$. These time frames make it difficult to explain the face advantage by faster detection at fixation only, since they allow for only a couple of additional fixations. Rather, we conclude that objects of expertise - and especially faces - must have a spatial, possibly parafoveal advantage.

\section{AUTHOR NOTE}

This research was supported by grants from the Israel Science Foundation and the U.S.-Israel Binational Science Foundation. O.H. was supported by a grant from the Hoffman Program for Social Leadership. We thank Aaron Elgavi and Tanya Orlov for helpful discussions during preparation of the manuscript, and Lior Kislev for use of bird photographs. The authors thank the anonymous reviewers for helpful suggestions. The authors declare that the research was conducted in the absence of any commercial or financial relationships that could be construed as a potential conflict of interest. Correspondence concerning this article should be addressed to O. Hershler, Neurobiology Department, Life Sciences Institute, Hebrew University, Givat Ram Campus, Jerusalem, Israel (e-mail: oritaron@gmail.com).

\section{REFERENCES}

Ahissar, M., \& Hochstein, S. (1997). Task difficulty and the specificity of perceptual learning. Nature, 387, 401-406.

Ahissar, M., \& HochsteIn, S. (2004). The reverse hierarchy theory of visual perceptual learning. Trends in Cognitive Sciences, 8, 457-464.

Burr, D. C., Morrone, M. C., \& Ross, J. (1994). Selective suppression of the magnocellular visual pathway during saccadic eye movements. Nature, 371, 511-513.

Carrasco, M., McLean, T. L., Katz, S. M., \& Frieder, K. S. (1998). Feature asymmetries in visual search: Effects of display duration, target eccentricity, orientation and spatial frequency. Vision Research, 38, 347-374.

CaVe, K. R., \& Wolfe, J. M. (1990). Modeling the role of parallel processing in visual search. Cognitive Psychology, 22, 225-271. 
Egeth, H. E., \& Yantis, S. (1997). Visual attention: Control, representation, and time course. Annual Review of Psychology, 48, 269-297.

Farah, M. J., TANaKa, J. W., \& Drain, H. M. (1995). What causes the face inversion effect? Journal of Experimental Psychology: Human Perception \& Performance, 21, 628-634.

Gauthier, I., Curran, T., Curby, K. M., \& Collins, D. (2003). Perceptual interference supports a nonmodular account of face processing. Nature Neuroscience, 6, 428-432.

GAUTHIER, I., \& TARR, M. J. (1997). Becoming a "greeble" expert: Exploring mechanisms for face recognition. Vision Research, 37, 1673 1682 .

GAUThIER, I., \& TARR, M. J. (2002). Unraveling mechanisms for expert object recognition: Bridging brain activity and behavior. Journal of Experimental Psychology: Human Perception \& Performance, 28, 431-446.

Goldstone, R. L. (2003). Learning to perceive while perceiving to learn. In R. Kimchi, M. Behrmann, \& C. R. Olson (Eds.), Perceptual organization in vision: Behavioral and neural perspectives (pp. 233278). Mahwah, NJ: Erlbaum.

Hershler, O., \& Hochstein, S. (2005). At first sight: A high-level pop out effect for faces. Vision Research, 45, 1707-1724.

Hershler, O., \& Hochstein, S. (2006). With a careful look: Still no low-level confound to face pop-out. Vision Research, 46, 3028-3035.

Hochstein, S., \& Ahissar, M. (2002). View from the top: Hierarchies and reverse hierarchies in the visual system. Neuron, 36, 791-804.

ITTI, L., \& KocH, C. (2000). A saliency-based search mechanism for overt and covert shifts of visual attention. Vision Research, 40, 14891506.

ItTI, L., \& KocH, C. (2001). Computational modeling of visual attention. Nature Reviews Neuroscience, 2, 194-203.

Johnson, K. E., \& Mervis, C. B. (1997). Effects of varying levels of expertise on the basic level of categorization. Journal of Experimental Psychology: General, 126, 248-277.

Koch, C., \& Ullman, S. (1985). Shifts in selective visual attention: Towards the underlying neural circuitry. Human Neurobiology, 4, 219-227.

Lee, D. K., Itti, L., Koch, C., \& Braun, J. (1999). Attention activates winner-take-all competition among visual filters. Nature Neuroscience, 2, 375-381.

Levine, D. N., \& Calvanio, R. (1989). Prosopagnosia: A defect in visual configural processing. Brain \& Cognition, 10, 149-170.

Malinowski, P., \& HüBnER, R. (2001). The effect of familiarity on visual-search performance: Evidence for learned basic features. Perception \& Psychophysics, 63, 458-463.

MruczeK, R. E. B., \& SheinberG, D. L. (2005). Distractor familiarity leads to more efficient visual search for complex stimuli. Perception \& Psychophysics, 67, 1016-1031

Palmeri, T. J., Wong, A. C.-N., \& Gauthier, I. (2004). Computational approaches to the development of perceptual expertise. Trends in Cognitive Sciences, 8, 378-386.

RAuschenberger, R., \& CHU, H. (2006). The effects of stimulus rotation and familiarity in visual search. Perception \& Psychophysics, 68, 770-775.

Reingold, E. M., Charness, N., Pomplun, M., \& Stampe, D. M. (2001). Visual span in expert chess players: Evidence from eye movements. Psychological Science, 12, 48-55.

Rosch, E., Mervis, C. B., Gray, W. D., Johnson, D. M., \& BoyesBraem, P. (1976). Basic objects in natural categories. Cognitive Psychology, 8, 382-439.

Shen, J., \& Reingold, E. M. (2001). Visual search asymmetry: The influence of stimulus familiarity and low-level features. Perception \& Psychophysics, 63, 464-475.

TANAKA, J. W., \& TAYLOR, M. (1991). Object categories and expertise: Is the basic level in the eye of the beholder? Cognitive Psychology, 23, 457-482.

TONG, F., \& NAKAYAMA, K. (1999). Robust representations for faces: Evidence from visual search. Journal of Experimental Psychology: Human Perception \& Performance, 25, 1016-1035.

Treisman, A. M., \& Gelade, G. (1980). A feature-integration theory of attention. Cognitive Psychology, 12, 97-136.

VANRullen, R. (2006). On second glance: Still no high-level pop-out effect for faces. Vision Research, 46, 3017-3027.

Wang, Q., Cavanagh, P., \& Green, M. (1994). Familiarity and pop-out in visual search. Perception \& Psychophysics, 56, 495-500.

Whiteley, A. M., \& Warrington, E. K. (1977). Prosopagnosia: A clinical, psychological, and anatomical study of three patients. Journal of Neurology, Neurosurgery, \& Psychiatry, 40, 395-403.

Wolfe, J. M., Butcher, S. J., Lee, C., \& Hyle, M. (2003). Changing your mind: On the contributions of top-down and bottom-up guidance in visual search for feature singletons. Journal of Experimental Psychology: Human Perception \& Performance, 29, 483-502.

(Manuscript received January 8, 2009; revision accepted for publication April 9, 2009.) 